Artículos de Psicología 



\title{
Prevalencia de estilos de afrontamiento y trastornos de somatización en docentes universitarios de dos facultades de psicología en Ibagué (Colombia)
}

\section{Prevalence of coping styles and somatization disorders in two university teaching psychology faculties in Ibague (Colombia)}

\author{
Luz Adriana Rubio' \\ Juan Sebastián Villarreal Moreno \\ Xatxia Charlenee Pérez Rodríguez \\ Margarita Rosa Guzmán García ${ }^{2}$
}

\begin{abstract}
Resumen
Objetivo. Identificar la posible influencia de los estilos de afrontamiento ante el estrés en el desarrollo del trastorno de somatización en docentes universitarios de tiempo completo de dos facultades de psicología de Ibagué (Tolima). Métodos. Se empleó el cuestionario de estrategias de afrontamiento frente al estrés, el Test de Othmer y DeSouza y la ficha sociodemográfica. Resultados. Del total de docentes que participaron en la investigación el $30 \%$ presenta tendencias a sufrir un posible trastorno de somatización. El Alfa de Cronbach de las escalas del CAE fue 0.959 (BAS), 0.778 (EEA), 0.762 (RLG), 0.807 (FSP), 0.580 (EVT), 0.356 (AFN) y -1.335 (REP). La estrategia más usada por los docentes fue: focalizado en la solución del problema con promedio de 3.35. De igual forma, se hallaron correlaciones positivas altas entre religión y evitación (0.870), religión y búsqueda de apoyo social $(0.763)$; reevaluación positiva y búsqueda de apoyo social (0.727); reevaluación positiva y evitación (0.778). Estos datos son importantes en la medida en que permiten visibilizar el estado de salud mental de los docentes universitarios y el uso de estrategias orientadas a la solución del problema que son fundamentales para mitigar la experiencia de estrés laboral en el contexto universitario.
\end{abstract}

Palabras clave: docencia universitaria, estrés laboral, psicología, trastornos de somatización.

\begin{abstract}
Objective. Identify the possible influence of coping styles to stress in the development of somatization disorder in college full-time teachers in two schools of psychology lbague (Tolima). Methods. Questionnaire coping strategies to stress, Othmer and DeSouza test, and sociodemographic record was used. Results. Of allteachers who participated in the research, 30\% present tendencies to suffer a possible somatization disorder. The Cronbach's alpha of the scales of the Styles Questionnaire to Cope with Stress (SQCS) was 0.959 (SSR), 0.778 (EEA), 0.762 (RLG), 0.807 (FSP), 0.580 (AVD), 0.356 (NST) and 1335 (PRE). The strategies used by teachers were: Focused on problem solving score of 3.35. High positive correlations between religion and avoidance (0.870), religion and social support (0.763) were found; positive reappraisal and
\end{abstract}

1 Docente Investigadora, Programa de Psicología. Universidad de San Buenaventura Medellín-Extensión Ibagué.

2 Estudiantes de Psicología. Universidad de San Buenaventura Medellín-Extensión Ibagué. 
social support (0.727); positive reappraisal and avoidance (0.778). This information is important as it allows us to visualize the mental health of college teachers and the use of oriented problem solving that are essential to mitigate the experience of occupational stress in the university context strategies.

Keywords: psychology, somatization disorders, university teaching, work stress.

Recibido: 19 de mayo de 2013 Aprobado: 16 de septiembre de 2013

La docencia es una labor importante en el desarrollo integral de quienes la ejercen. Esta trae consigo asumir funciones, responsabilidades $y$ retos que exigen esfuerzo y compromiso constante. Generalmente, el docente se ve expuesto a niveles altos de estrés, convirtiéndose en uno de los empleos con mayor riesgo psicosocial (Domínguez, 2004). El presente estudio busca hacer un recorrido de sus labores, considerando dos grandesámbitos:la orientación académica y la labor administrativa, donde las condiciones en este contexto laboral pueden resultar en experiencias de fracaso, frustración y malestar, ocasionando consecuencias nocivas para la salud física y mental del docente (Marulanda, 2007).Por tanto, es importante tipificar los posibles síntomas somatomorfos en docentes universitariosde tiempo completo de dos facultades de psicología de Ibagué, para así determinar la relación entre un posible estrés laboral y la emergencia de síntomas somáticos, al tiempo que se pretende identificar sus estilos de afrontamiento de situaciones de tensión, los cuales pueden estar relacionados con el problema o con la emoción, siendo este último un factor de riesgo psicosocial.

El estrés laboral, más conocido hoy día como síndrome de burnout, está caracterizado por un conjunto de síntomas médico-biológicos y psicosociales, donde el paciente presenta una progresiva pérdida de energía, llegando al agotamiento pues aquellos tanto físico como psicológico, y presentando síntomas de ansiedad, depresión, desmotivacióneneltrabajo,agresividad e irritabilidad (Freudenberg; citado en Quintanilla, 2004). En esta línea, en los años ochenta, autores como Susan Jackson y Cristina Maslach centran su atención en profesionales de agencias de servicio público, pues aquellos definen sus creencias, expectativas $u$ opiniones esperando coincidir con las de su empresa. De esta manera, cuando existe una incongruencia, puede generarse una insatisfacción personal hacia el trabajo, la cual llegaría a desencadenar el síndrome de "estar quemado" (Carrillo, Gómez y Espinosa, 2012). Inicialmente, Maslach y Jackson (citados en GilMonte,2003), desde una perspectiva psicosocial, plantean tres dimensiones: a) agotamiento emocional, que consiste en una deficiencia en los recursos emocionales propios, percibiéndose como incapaces de dar más de sí mismos a nivel afectivo, debido al contacto diario con personas a las que se atiende; b) despersonalización, es la respuesta excesivamente negativa, insensible y despreocupadahaciaotraspersonas, caracterizada por actitudes de cinismo, sentimientos de impotencia, indefensión, desesperanza personal y endurecimiento afectivo, con lo que se ve a las personas de forma deshumanizada, culpándolas de sus problemas, y c) falta de autorrealización personal, es decir, deterioro de los propios sentimientos de competencia y éxito, afectando la realización de su trabajo y la relación con compañeros, asociado con lapérdida de interés en sus ideales, alejamiento de actividades familiares y sociales(Guerrero, 2003).

El síndrome de burnout suele ocurrir entre individuos que trabajan con un número amplio de personas, que demandan gran cantidad de atención para resolver múltiples problemas a la vez, además de coordinación de actividades que no solo dependen de un solo empleado, sino de un trabajo en equipo que debe ser organizado, sincronizado, comprometido y competente. Al mismo tiempo, las tareas se dificultan por problemas de comunicación 
y estilos de desempeño laboral, ya que cada empleado tiene un ritmo y una manera distinta de operar (Carrillo, Gómez y Espinosa, 2012). Del mismo modo, al considerar las posibles causas que precipitan el desarrollo del estrés laboral en docentes, debe tenerse en cuenta varios factores, como los personales y los ambientales: la dimensión personal, para el surgimiento del síndrome del burnout, puede estar relacionada con las rasgos de personalidad propensos a desarrollar alteraciones emocionales que afectan significativamente su labor profesional, especialmente cuando se atiende a otras personas durante espacios prolongados de tiempo (Matud, García y Matud, 2002). En efecto, para que una situación sea potencialmente estresante, las características personales entran a desempeñar un papel importante. De esta forma, Lazarus (1999, citado por Casado,2000) define estas características según las metas y su jerarquía, las creencias acerca de sí mismo y del mundo, así como los recursos personales con que cuentan los seres humanos para responder ante una situación desafiante.

Por ende, los procesos cognoscitivos, emocionales y conductuales influyen en la forma como se enfrenta y maneja un evento estresante. Así, la evaluación cognitiva es la que finalmente determina que una relación individuo-ambiente resulte estresante o no (Solis y Vidal, 2006). Consecuentemente, el afrontamiento es entendido como los esfuerzos cognoscitivos y conductuales que se desarrollan para manejar el estrés y reducir las tensiones causadas por las situaciones aversivas, tanto internas como externas, que las personas enfrentan en el curso de la vida (Casado, 2000). Por otra parte, la praxis del profesional en psicología, especialmente los educativos que se enfrentan ante diferentes retos psicosociales, ponen a prueba su capacidad cognitiva y versatilidad a la hora de emplear procedimientos de intervención psicoeducativa, y técnicas de evaluación e intervención clínica, tales como el psicodiagnóstico, terapia y modificación de conducta de los estudiantes para facilitar su rendimiento académico (Sánchez, Mardones, 2010).
Equitativamente, el psicólogo se ve atrapado en dos condiciones psicoafectivas patológicas: una clínica (signos y síntomas psicopatológicos) y otra psicosocial (Gil-Monte y Peiró, 1997 citado en Guerrero, 2003). En la primera el individuo en condición de burnout experimenta estrés laboral, presentando dificultades notables para lograr un equilibrio emocional; mientras, la segunda es el resultado de un proceso de desajuste personal y social que se desarrolla por la interacción inadecuada en su entorno laboral. Generalmente, el psicólogo que trabaja en el área educativa se ve expuesto a situaciones de estrés, tales como: el ambiente laboral inadecuado, sobrecarga de trabajo y largas jornadas que superan las ocho horas reglamentarias, que en muchos casos generan alteración de ritmos biológicos, responsabilidades y decisiones importantes (Ponceet al., 2005).Igualmente, implica asumir diversas funciones y complejas tareas asignadas en espacios de tiempo limitados (Flores y cols., 2009), además de compromisos laborales paralelos, ya que los psicólogos trabajan para varias instituciones a la vezcomo catedráticos, atienden consultorios particulares o desarrollan proyectos con entidades del Estado que demandan bastante tiempo y dedicación (Carrasco y Patricio, 2012). Del mismo modo, las relaciones interpersonales conflictivas con compañeros de trabajo y superiores de manera frecuente se convierten en foco de tensión y malestar psicológico para el docente (Matud, García y Matud, 2002).

De este modo, al revisar la incidencia del estrés laboral en el personal docente, se ha encontrado repercusiones en la salud, desencadenando problemas de somatización, el cual es considerado como una serie de procesos patológicos agrupados según el DSM-IV como trastornos somatomorfos, caracterizados por "la presencia de síntomas corporales que sugieren un trastorno físico sin causa orgánica demostrable o mecanismo fisiológico conocido que los explique completamente y por la presunción razonable de que dichos síntomas están asociados a factores psicológicos o estrés" (Guzmán, 2011). 


\section{Método}

Esta es una investigación cuantitativa de tipo explicativo con un diseño descriptivocorrelacional, de corte empírico-analítico, con la participación voluntaria y anónima de diez docentes de tiempo completo de dos facultades de Psicología de Ibagué. Se implementan instrumentos como el Cuestionario de estrategias de afrontamiento frente al estrés (CAE),que es una medida de autoinforme diseñada para evaluar siete estilos de afrontamiento, divididos en dos grupos: uno orientado hacia el problema y el otro enfocado en la emoción. Este cuestionario fue elaborado por Lazarus y Folkman (1986). Por otro lado, se aplica el test de Othmer y DeSouza, que es una escalapara el screening del trastorno de somatización, con el fin de identificar síntomas fisiológicos que pueden estar relacionados con alteraciones en el estado del ánimo, especialmente la ansiedad. Por último, se implementa la ficha de caracterización, con el objetivo de visualizar en la población aspectos de interés, como su estado civil, tipo de familia, estudios superiores, vinculación laboral y algunas preguntas relacionadas con la satisfacción laboral. Posteriormente, para el análisis de los datos se empleó el software estadístico IBM SPSS stastistics 2.0. Cabe resaltar que los docentes recibieron los instrumentos y el consentimiento informado que contienenlos principios bioéticos de la investigación.Finalmente, las variables que se tomaron en cuenta fueron las siguientes: respecto al cuestionario de estilos de afrontamiento, el focalizado en la solución del problema, autofocalización negativa, reevaluación positiva, expresión emocional abierta, evitación, búsqueda de apoyo socialy religión. Del mismo modo, del test de Othmer y DeSouza se tomó como referencia: dolor corporal, sensación de quemazón, vómitos frecuentes y dificultades para respirar.

\section{Resultados}

De los docentes universitarios que trabajan de tiempo completo en las dos facultades de psicología seleccionadas para el presente estudio, se encontró que la edad promedio es de 37.9 años; en otras palabras, el $30 \%$ tiene entre 39 a 42 . El $60 \%$ de los docentes son mujeres, el $40 \%$ son solteros, el $40 \%$ son casados y el $20 \%$ son divorciados. El $70 \%$ pertenecen al estrato socioeconómico 3 . El $60 \%$ no vive en casa propia. El $50 \%$ viven con 3 a 4 personas, $10 \%$ viven con 1 a 2 , otro $10 \%$ con más de 5 personas y el $30 \%$ viven solos. El $60 \%$ tiene entre 1 y 2 hijos y el $40 \%$ no tiene. El número de personas a cargo esde 1 a 2 personas (30\%), 3 a 4 (20\%) y el $50 \%$ no tiene. El tipo de familias conformadas son: el $60 \%$ nuclear, 30\% monoparental y $10 \%$ extensa. En cuanto a sus estudios, el $40 \%$ tiene especialización, el 30\% maestría, el 30\% doctorado. De las personas que viven con los docentes, actualmente trabajan de 1 a 2 personas en el $20 \%$ de los casos y en el $80 \%$ no lo hace.

Todos docentes de del estudio son psicólogos y el $20 \%$ ejerce en el campo educativo, otro $20 \%$ en el clínico, un $10 \%$ en el organizacional, el $30 \%$ en tres campos ocupacionales y el último $20 \%$ dos campos. De ellos el $70 \%$ trabaja en 3 y 4 entidades distintas y el $30 \%$ en 1 y 2 . El $30 \%$ realiza actividades tanto académicas como administrativas y ninguno hace atención clínica en la institución educativa. El $70 \%$ de los docentes le dedica a la universidad entre 31 a 60 horas semanales y el 10\% más de 61 horas. El 40\% de los docentes posee consultorio particular y un $10 \%$ le dedica cuatro horas semanales, $20 \%$ seis horas y otro $10 \%$. Cuando se les preguntó a los docentes cuántas horas duermen diariamente, el $80 \%$ respondió que entre 5 y 6 horas, el $10 \%$ de 3 a 4 y otro $10 \%$ de 1 a 2 horas. Un $40 \%$ de los docentes dedican a sus familias entre 1 y 2 horas, un $30 \%$ entre 3 y 4 horas, $10 \%$ entre 5 y 6 horas, y el $20 \%$ no dedican tiempo. Un 40\% la última vez que realizó un viaje familiar fue hace un mes; varios meses un $30 \%$, menos de un año un $10 \%$, y más de un año, $20 \%$.

\section{Screening del trastorno de somatización}

Del total de docentes que participaron en la investigación, el $30 \%$ presenta tendencias a sufrir un posible trastorno de somatización, frente a un $70 \%$ que no lo posee. 
Tabla 1. Estadístico test de Othmer y DeSouza

\begin{tabular}{|c|c|c|}
\hline $\begin{array}{c}\text { Screening del trastorno de } \\
\text { somatización }\end{array}$ & $\mathbf{N}^{\circ}$ & $\%$ \\
\hline $\begin{array}{c}\text { Sin trastorno de somatización } \\
\text { Posible trastorno de } \\
\text { somatización }\end{array}$ & 7 & 70.0 \\
\hline
\end{tabular}

Con las variables de estudio: búsqueda de apoyo social (BAS), expresión emocional abierta (EEO), religión (RLG), focalización en la solución del problema (FSP), evitación (EVT), autofocalización negativa (AFN), reevaluación positiva (REP), contempladas en la aplicación del cuestionario $C A E$, se hallaron los siguientes datos:

Tabla 2. Factor: 1 Búsqueda de apoyo social (BAS)

\begin{tabular}{|l|c|c|c|}
\hline \multicolumn{2}{|c|}{ ÍTEM } & Media & $\begin{array}{c}\text { Desviación } \\
\text { típica }\end{array}$ \\
\hline $\begin{array}{l}\text { Le conté a } \\
\text { familiares o } \\
\text { amigos cómo me } \\
\text { sentía }\end{array}$ & 2.60 & 1.174 & 10 \\
\hline $\begin{array}{l}\text { Pedí consejo a } \\
\text { algún pariente } \\
\text { o amigo para } \\
\text { afrontar mejor el } \\
\text { problema }\end{array}$ & 2.30 & 1.418 & 10 \\
\hline $\begin{array}{l}\text { Pedí a parientes } \\
\text { o amigos que } \\
\text { me ayudaran a } \\
\text { pensar acerca del } \\
\text { problema }\end{array}$ & 2.40 & 1.350 & 10 \\
\hline $\begin{array}{l}\text { Hablé con amigos } \\
\text { o familiares } \\
\text { para que me } \\
\text { tranquilizaran } \\
\text { cuando me } \\
\text { encontraba mal }\end{array}$ & 2.40 & 1.578 & 10 \\
\hline $\begin{array}{l}\text { Pedí a algún } \\
\text { amigo o familiar } \\
\text { que me indicara } \\
\text { cuál sería el mejor } \\
\text { camino a seguir }\end{array}$ & 1.90 & 1.370 & 10 \\
\hline $\begin{array}{l}\text { Procuré que algún } \\
\text { familiar o amigo } \\
\text { me escuchase } \\
\text { cuando necesité } \\
\text { manifestar mis } \\
\text { sentimientos }\end{array}$ & 2.30 & 1.337 & 10 \\
\hline \begin{tabular}{l} 
Media total \\
\hline
\end{tabular} & 2.32 & 1.372 & 10 \\
\hline
\end{tabular}

Tabla 3. Factor 2: Expresión emocional abierta (EEA)

\begin{tabular}{|l|c|c|c|}
\multicolumn{1}{|c}{ ÍTEM } & \multicolumn{1}{c|}{ Media } & \multicolumn{1}{c|}{$\begin{array}{c}\text { Desviación } \\
\text { típica }\end{array}$} & Núm. \\
\hline $\begin{array}{l}\text { Descargué mi mal } \\
\text { humor con los } \\
\text { demás }\end{array}$ & 1.50 & 0.850 & 10 \\
$\begin{array}{l}\text { Insulté a ciertas } \\
\text { personas }\end{array}$ & 0.50 & 0.850 & 10 \\
$\begin{array}{l}\text { Me comporté de } \\
\text { forma hostil con } \\
\text { los demás }\end{array}$ & 0.70 & 0.823 & 10 \\
$\begin{array}{l}\text { Agredí a algunas } \\
\text { personas }\end{array}$ & 0.10 & 0.316 & 10 \\
$\begin{array}{l}\text { Me irrité con } \\
\text { alguna gente }\end{array}$ & 1.30 & 1.160 & 10 \\
$\begin{array}{l}\text { Luché y me } \\
\text { desahogué } \\
\text { expresando mis } \\
\text { sentimientos } \\
\text { Media total }\end{array}$ & 2.90 & 1.197 & 10 \\
\hline
\end{tabular}

Tabla 4. Factor 3: Religión (RLG)

\begin{tabular}{|l|c|c|c|}
\hline \multicolumn{1}{|c|}{ ÍTEM } & \multicolumn{1}{c|}{ Media } & \multicolumn{1}{c|}{$\begin{array}{c}\text { Desviación } \\
\text { típica }\end{array}$} & Núm. \\
\hline Asistí a la iglesia & 1.90 & 1.663 & 10 \\
\hline $\begin{array}{l}\text { Pedí ayuda } \\
\text { espiritual ra } \\
\text { algún religioso } \\
\text { (sacerdote, etc.) }\end{array}$ & 0.60 & 1.265 & 10 \\
\hline $\begin{array}{l}\text { Acudí a la iglesia } \\
\text { para rogar que } \\
\text { se solucionase el } \\
\text { problema }\end{array}$ & 1.70 & 1.636 & 10 \\
\hline $\begin{array}{l}\text { Tuve fe en que } \\
\text { Dios remediaría la } \\
\text { situación }\end{array}$ & 2.10 & 1.663 & 10 \\
\hline Recé & 2.50 & 1.434 & 10 \\
\hline $\begin{array}{l}\text { Acudí a la iglesia } \\
\text { para poner velas } \\
\text { o rezar }\end{array}$ & 1.30 & 1.337 & 10 \\
\hline Media total & 1.68 & 1.500 & 10 \\
\hline
\end{tabular}


Tabla 5. Factor 4: Focalizado en la solución del problema (FSP)

\begin{tabular}{|c|c|c|c|}
\hline ÍTEM & Media & $\begin{array}{l}\text { Desviación } \\
\text { típica }\end{array}$ & Núm. \\
\hline $\begin{array}{l}\text { Traté de analizar } \\
\text { las causas del } \\
\text { problema para } \\
\text { poder hacerle } \\
\text { frente }\end{array}$ & 3.60 & 0.516 & 10 \\
\hline $\begin{array}{l}\text { Traté de } \\
\text { solucionar } \\
\text { el problema } \\
\text { siguiendo unos } \\
\text { pasos bien } \\
\text { pensados }\end{array}$ & 3.40 & 0.699 & 10 \\
\hline $\begin{array}{l}\text { Establecí un plan } \\
\text { de actuación y } \\
\text { procuré llevarlo a } \\
\text { cabo }\end{array}$ & 3.20 & 0.789 & 10 \\
\hline $\begin{array}{l}\text { Hablé con } \\
\text { las personas } \\
\text { implicadas } \\
\text { para encontrar } \\
\text { una solución al } \\
\text { problema }\end{array}$ & 3.20 & 0.632 & 10 \\
\hline $\begin{array}{l}\text { Hice frente } \\
\text { al problema } \\
\text { poniendo en } \\
\text { marcha varias } \\
\text { soluciones } \\
\text { concretas }\end{array}$ & 3.30 & 0.949 & 10 \\
\hline $\begin{array}{l}\text { Pensé } \\
\text { detenidamente } \\
\text { los pasos a seguir } \\
\text { para enfrentarme } \\
\text { al problema }\end{array}$ & 3.40 & 0.516 & 10 \\
\hline Media total & 3.35 & 0.684 & 10 \\
\hline
\end{tabular}

Tabla 6. Factor 5: Evitación (EVT)

\begin{tabular}{|l|c|c|c|}
\multicolumn{1}{|c}{ ÍTEM } & \multicolumn{1}{c|}{ Media } & \multicolumn{1}{c|}{$\begin{array}{c}\text { Desviación } \\
\text { típica }\end{array}$} & Núm. \\
\hline $\begin{array}{l}\text { Cuando me venía } \\
\text { a la cabeza el } \\
\text { problema, trataba } \\
\text { de concentrarme } \\
\text { en otras cosas }\end{array}$ & 2.40 & 1.350 & 10 \\
\hline $\begin{array}{l}\text { Me volqué en el } \\
\text { trabajo o en otra } \\
\text { actividad para } \\
\text { olvidarme del } \\
\text { problema }\end{array}$ & 2.50 & 1.179 & 10 \\
\hline
\end{tabular}

Viene de la colúmna anterior...

\begin{tabular}{|l|c|c|c|}
\hline \multicolumn{1}{|c|}{ ÍTEM } & \multicolumn{1}{c|}{ Media } & $\begin{array}{c}\text { Desviación } \\
\text { típica }\end{array}$ & Núm. \\
\hline $\begin{array}{l}\text { Salí a cine, a } \\
\text { cenar, a"dar una } \\
\text { vuelta", etc. Para } \\
\text { olvidarme del } \\
\text { problema }\end{array}$ & 1.70 & 1.337 & 10 \\
\hline $\begin{array}{l}\text { Procuré no pensar } \\
\text { en el problema }\end{array}$ & 1.30 & 1.059 & 10 \\
\hline $\begin{array}{l}\text { Practiqué algún } \\
\text { deporte para } \\
\text { olvidarme del } \\
\text { problema }\end{array}$ & 1.40 & 1.350 & 10 \\
\hline $\begin{array}{l}\text { Intenté olvidarme } \\
\text { de todo }\end{array}$ & 1.00 & 0.816 & 10 \\
\hline Media total & 1.72 & 1.182 & 10 \\
\hline
\end{tabular}

Tabla 7. Factor 6: Autofocalizaciónnegativa (AFN)

\begin{tabular}{|l|l|l|l|}
\hline \multicolumn{1}{|c|}{ ÍTEM } & Media & \multicolumn{1}{c|}{$\begin{array}{c}\text { Desviación } \\
\text { típica }\end{array}$} & Núm. \\
\hline $\begin{array}{l}\text { Me convencí de } \\
\text { que hiciera lo } \\
\text { que hiciera las } \\
\text { cosas siempre me } \\
\text { saldrían mal }\end{array}$ & 0.60 & 0.966 & 10 \\
\hline $\begin{array}{l}\text { No hice nada } \\
\text { concreto puesto } \\
\text { que las cosas } \\
\text { suelen ser malas }\end{array}$ & 0.50 & 0.707 & 10 \\
\hline $\begin{array}{l}\text { Comprendí que } \\
\text { yo fui el principal } \\
\text { causante del } \\
\text { problema } 2.10\end{array}$ & 1.197 & 10 \\
\hline $\begin{array}{l}\text { Me sentí } \\
\text { indefenso/a e } \\
\text { incapaz de hacer } \\
\text { algo positivo } \\
\text { para cambiar la } \\
\text { situación }\end{array}$ & 0.50 & 0.850 & 10 \\
\hline $\begin{array}{l}\text { Me di cuenta de } \\
\text { que por mí mismo } \\
\text { no podía hacer } \\
\text { nada para resolver } \\
\text { el problema }\end{array}$ & 0.60 & 1.265 & 10 \\
\hline $\begin{array}{l}\text { Me resigné a } \\
\text { aceptar las cosas } \\
\text { como eran }\end{array}$ & 0.80 & 1.135 & 10 \\
\hline Media total & 0.85 & 1.020 & 10 \\
\hline
\end{tabular}


Tabla 8. Factor7: Reevaluación positiva (REP)

\begin{tabular}{|l|c|c|c|}
\hline \multicolumn{2}{|c|}{ ÍTEM } & Media & \multicolumn{1}{c|}{$\begin{array}{c}\text { Desviación } \\
\text { típica }\end{array}$} \\
\hline $\begin{array}{l}\text { Intenté } \\
\text { centrarme en } \\
\text { los aspectos } \\
\text { positivos del } \\
\text { problema }\end{array}$ & 3.10 & 0.738 & 10 \\
\hline $\begin{array}{l}\text { Intenté sacar } \\
\text { algo positivo del } \\
\text { problema }\end{array}$ & 3.60 & 0.516 & 10 \\
\hline $\begin{array}{l}\text { Descubrí que en } \\
\text { la vida hay cosas } \\
\text { buenas y gente } \\
\text { que se preocupa } \\
\text { por los demás }\end{array}$ & 3.00 & 1.247 & 10 \\
\hline $\begin{array}{l}\text { Comprendí que } \\
\text { otras cosas, } \\
\text { diferentes del } \\
\text { problema, eran } \\
\text { para mí más } \\
\text { importantes }\end{array}$ & 2.70 & 1.337 & 10 \\
\hline $\begin{array}{l}\text { Experimenté } \\
\text { personalmente } \\
\text { eso de que "no } \\
\text { hay mal que por } \\
\text { bien no venga" }\end{array}$ & 1.50 & 1.434 & 10 \\
\hline $\begin{array}{l}\text { Comprobé que, } \\
\text { después de todo, } \\
\text { las cosas podían } \\
\text { haber ocurrido } \\
\text { peor }\end{array}$ & 2.20 & 1.229 & 10 \\
\hline
\end{tabular}

\section{Correlación de Pearson}

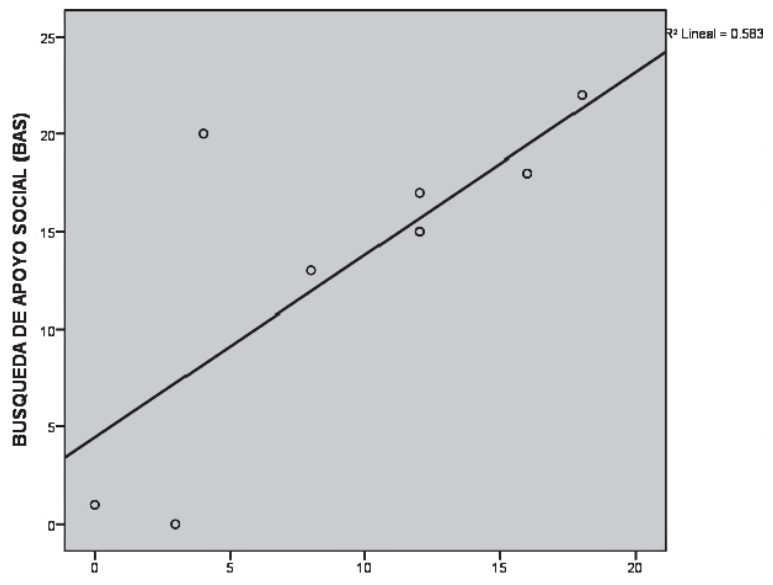

Figura 1: Correlación de Pearson (BAS-RLG)

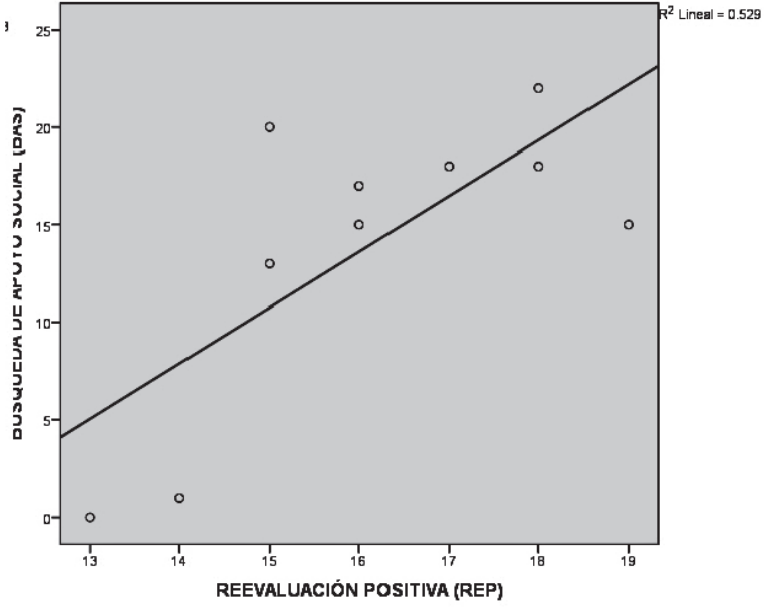

Figura2: Correlación de Pearson (BAS-REP)

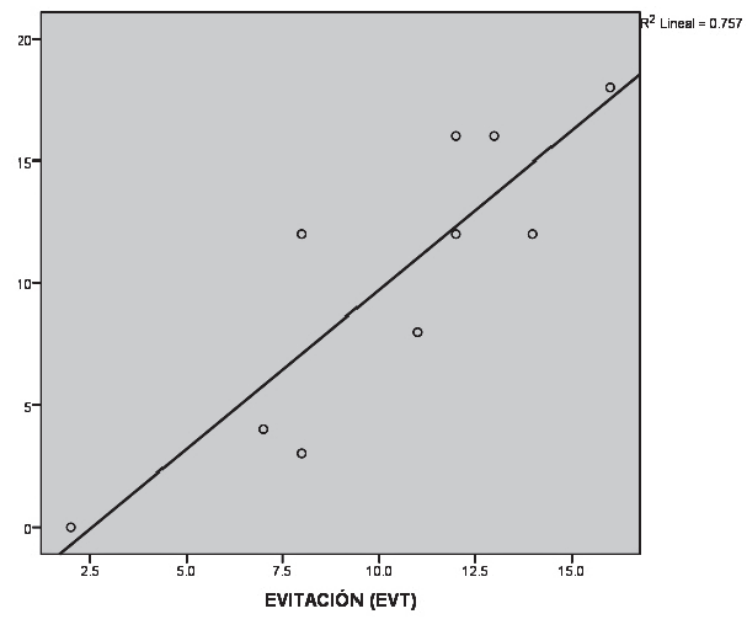

Figura3: Correlación de Pearson (BAS-EVT)

Se hallaron correlaciones positivas altas entre religión y evitación $(0.870, \mathrm{p}=0.001)$; religión y búsqueda de apoyo social (0.763, $\mathrm{p}=.010)$; reevaluación positiva y búsqueda de apoyo social (0.727, $\mathrm{p}=0.017)$; reevaluación positiva y evitación (0.778, $\mathrm{p}=0.008)$. Por el contrario, al hacer la correlación entre la variable de somatización y las variables del CAE se encontraron correlaciones bajas ensomatización y búsqueda de apoyo social $(0.195, p=0.590)$; somatización y expresión emocional abierta (0.381, $\mathrm{p}=0.043)$. Además, se encontró que los valores de $p$ son superiores a 0.05 (5\%), concluyéndose que no existe correlación entre somatización y religión $(0.195$, $\mathrm{p}=0.599$ ), así como somatización y focalización 
en la solución del problema $(0.138, \mathrm{p}=0.704)$, somatización y evitación (-0.202, $\mathrm{p}=0.575)$, somatización y autofocalización negativa $(0.033$, $\mathrm{p}=0.928)$ y las de somatización y reevaluación positiva $(0.085, \mathrm{p}=0.815)$.

\section{Discusión}

Teniendo en cuenta los resultados con la aplicación del test de Othmer y DeSouza, se pudo observar que un porcentaje importante (30\%)de docentes universitarios afirmaron haber padecido síntomas relacionados con el trastorno de somatización, lo cual reafirma la idea de que la docencia es una de las profesiones con mayor riesgo psicosocial (Pandoet al., 2006). Sin embargo, resulta interesante el resultado de hacer el contraste entre las variables de somatización y las del $\mathrm{CAE}$,con lo que se encontraron correlaciones en somatización y búsqueda de apoyo social (0.195); somatización y expresión emocional abierta (0.381). Además, se encontraron correlaciones muy bajas entre somatización y religión (0.195), así como somatización y focalización en la solución del problema (0.138). Llama la atención que entre somatización y evitación se halló una correlación negativa baja (-0.202), lo que quiere decir que cuando los síntomas relacionados con el trastorno de somatización disminuyen, existe una tendencia mayor a desarrollar conductas de evitación. En este sentido, al alejarse de los eventos estresores sin hacerle frente de manera directa incide favorablemente en la reducción de experiencias de malestar corporal sin causa orgánica aparente.

No obstante, a pesar de que solo el $30 \%$ de los docentes manifestaron estar presentando síntomas relacionados con dolencias corporales no tipificados dentro de una enfermedad fisiológica determinada, se demuestra que la somatización está asociada con el estrés. De hecho, González y Landero (2008) demuestran que los trastornos y síntomas psicosomáticos pueden relacionarse con múltiples factores, entre los que se encuentran el estrés. En elestudio de Guzmán (2011) se encuentraque los trastornos somatomorfos constituyen un cuadropatológico, cuyo diagnóstico se realiza por la presencia de cambios corporales que sugieren una alteración física sin causa orgánica o determinada que los explique completamente.

Continuando con el análisis de los resultados del $C A E$, se observa que en el Factor 1: búsqueda de apoyo social, se obtuvo una media en las respuestas (2.32), lo cual indica que los docentes respondieron en su mayoría a las preguntas de esta variable con la opción: A veces. Esta dimensión está relacionada con la estrategia de hacer uso de sus redes de apoyo familiar y social para encontrar conjuntamente soluciones a sus dificultades y así poder afrontar mejor el problema. Esta respuesta permite observar que los docentes no siempre suelen depender de otras opiniones para afrontar las situaciones difíciles, es decir que tienden a ser más autónomos. Esto puede corroborarse al observar el Factor 4: focalizado en la solución del problema, que puntúo en promedio 3.35, equivalente a quefrecuentemente tienden a centrarse en la resolución del conflicto, tratando de analizar las causas para poder hacerle frente, de forma sistemática con las personas implicadas, procurando encontrar una rápida y efectiva solución al problema y poniendo en marcha diferentes alternativas. Otra estrategia muy usada es la del Factor 7: reevaluación positiva, que puntuó 3 en promedio de respuesta, que corresponde a que frecuentemente tienden a centrarse en los aspectos positivos del problema, asumiendo que en la vida la mejor alternativa es ser optimista frente a las situaciones y las personas con las que se convive y comparte diariamente. De este modo, las estrategias más utilizadas por los docentes son: Búsqueda de apoyo social, focalizado en la solución del problema y reevaluación positiva; similar a las estrategias usadas por los docentes de una universidad mexicana según el estudio realizado por Valadez, Bravo y Vaquero (2011: 71), quienes reportaron usar de manera frecuente las estrategias de "Planificación 55.6\%, Reevaluación Positiva 52.4\%, Autocontrol 48.4\%, Búsqueda de Apoyo Social 44.9\%, Confrontación 19.1\%, 
Distanciamiento $17.5 \%$, por último, EvitaciónHuida 10.3\%". Tales resultados muestran una especie de protección, usada por los docentes que participaron en la presente investigación, evitando evaluar las situación como desbordantes de la capacidad del individuo para resolverlas; así, algunos estilos de afrontamiento se utilizan para minimizar el impacto que producen los estresores sobre el estado psicológico y físico de las personas (Contreras, Espinosa y Esquerra, 2007).

Después de explorar los resultados de las variables más representativas, es pertinente examinar las correlaciones positivas altas entre religión y evitación (0.870); religión y búsqueda de apoyo social (0.763); reevaluación positiva y búsqueda de apoyo social (0.727); reevaluación positiva y evitación (0.778). A pesar de que los docentes tienden a mostrar una posición racional basada en lo científico, expresando que poseen la habilidad para controlar las situaciones y poder afrontar desafíos personales, familiares y profesionales, lo cierto es que implícitamente reflejan una importante espiritualidad asociada de manera congruente con las respuestas de evasión, pues dejan en manos de un ser divino la solución de ciertos problemas que son percibidos como desbordantes. Este estilo de afrontamiento está más orientado hacia la emoción, según lo propuesto por Lazarus y Folkman (1986), donde el sujeto no modifica la situación por considerar que no es posible resolverlo, evitando afrontar la situación problemática. Este es un estilo con el que la persona opta por darle un manejo noreal o ilusorio a la situación, por lo que en algunos casos se puede interpretar como manipulativo, por atribuir la culpa a otros, negando su propia responsabilidad. Por otro lado, llama la atención la alta correlación entre reevaluación positiva y búsqueda de apoyo social, ya que es un poco contradictorio con lo descrito. La reevaluación positiva se refiere a la tendencia de los docentes a centrarse en los aspectos positivos del problema, sumado a la necesidad de buscar apoyo en familiares y amigos para asegurarse de tomar la mejor decisión; estas estrategias coinciden con lo hallado en el estudio realizado por Valadez, Bravo y Vaquero (2011), donde los docentes tienden a buscar apoyo social, dado que interactúan socialmente con mayor facilidad.

También, es fundamental revisar los resultados de las variables que tuvieron puntuaciones bajas como la expresión emocional abierta, con una media de respuesta de 1.17, que equivale a que pocas veces los docentes descargan su mal humor con los demás, insultándolos o comportándose de forma hostil, mostrando conductas agresivas e irritables o expresando de manera abierta sus sentimientos. Según este hallazgo, lo que permite inferir es que los docentes son personas que suelen controlar sus emociones e impulsos, al tener clara su posición y el rol que cumplen dentro la institución educativa. Otro que pertenece al estilo orientado hacia la emoción es el Factor 5: evitación, en el que los docentes respondieron con un 1.72en promedio; en otras palabras, pocas veces los docentes tienden a concentrarse en otras actividades, como refugiarse en el trabajo, salir a cine, a cenar, a dar una vuelta o practicar algún deporte, para olvidar el problema con el fin de evitarlo. Estos resultados pueden darse debido a las herramientas con que cuenta el docente, por ser psicólogo, al ser un profesional que cuenta con los conocimientos suficientes en entrenamiento de habilidades sociales, resolución de conflictos, asertividad, entre otras estrategias psicológicas.

Con todo, González y Landero (2008) proponen un modelo explicativo del estrés y de los síntomas psicosomáticos, afirmando que una persona, cuando percibe algún nivel de estrés, puede provocar respuestas emocionales, conductuales y fisiológicas.

Al examinarlos datos sociodemográficos, se pudo identificar aspectos importantes, como que la edad promedio de docentes universitarios que laboran tiempo completo es de 37.9 años, indicando así que se encuentran en la etapa de la adultez temprana, periodo en el que las personas "hacen elecciones de vida significativas y exhiben la mayor energía pero también experimenta el mayor estrés" (Fernández, 2006). Asimismo, es importante revisar el género y el estado civil, 
ya que estos permiten correlacionar el factor de riesgo para presentar síndrome de burnout. En una investigación realizada por Palmeret al. (2005) se afirma que el ser mujer, soltero y no tener hijos facilita sufrir de estrés laboral. En esta investigación se halló que la mayoría son mujeres(60\%), el $40 \%$ son solteros, $40 \%$ casados y $20 \%$ divorciados; tipo de familia conformada en su mayoría nuclear (60\%). En este sentido los resultados están bastantes parcializados entorno al estado civil, pero vale la pena enfatizar en la importancia de tener el apoyo familiar para mitigar los efectos de la presión laboral, además de ser un factor regulador del tiempo que se le dedica al trabajo.

En cuanto al estrato socioeconómico, la mayoría (70\%) pertenecen al 3 y viven enalquiler $(60 \%)$, con 3 a 4 personas, tiene entre 1 y 2 hijos (60\%), con edades entre los 6 y los 10 (30\%). El número de personas a cargo son entre 1 a 2 (30\%). Con relación a estos datos se puede revisar los efectos de adquirir responsabilidades económicas al tener más de dos o tres personas a cargo, lo que exigen tener más de dos empleos para poder asumir los gastos del hogar. Este puede ser un factor desencadenante del síndrome de burnout, debido a la excesiva demanda laboral (Mamaniet al., 2007).

En cuanto a sus estudios, el $40 \%$ tiene especialización, 30\% maestría y 30\% doctorado, lo que se considera como un factor protector en la medida en que a mayor formación académica, mayores son las herramientas con las que se cuentan para enfrentar situaciones desafiantes en la vida, además de que brinda mayor satisfacción personal, producto de su posicionamiento laboral, que lo perfila como un profesional competente (Vegaet al., 2009). El 20\% son psicólogos y ejerce el campo educativo, 20\% clínico, $10 \%$ organizacional, $30 \%$ ejercen tres campos y el $20 \%$ dos campos. Llama la atención las cifras tan altas de docentes que ejercen tres campos a la vez, debido a que esta labor exige del profesional mayor esfuerzo cognitivo, emocional y comportamental, llevándolo a enfrentar una posible sobrecarga laboral, responsable del desarrollo del síndrome de burnout, conocido ampliamente como una situación crónica de estrés, según lo que plantea Moriana y Herruzo (2004).Además, es importante considerar que otro de los factores que hace posible que los docentes experimenten estrés es el número de instituciones para las que se labora. En efecto, se observó que de los docentes encuestados el $70 \%$ trabaja en 3 y 4 entidades distintas y el $30 \%$ en 1 y 2 . Generalmente los docentes manifiestan que se dedican a la docencia por vocación, pero esta se ve afectada por las otras responsabilidades adquiridas, reduciendo el tiempo que debe dedicarse a la academia y a la investigación. Es por ello que la docencia puede, en muchas ocasiones, ser ambivalente, ya que según lo dicho por la Federación de enseñanza CC.OO. de Andalucía (2012)“por un lado, existe un fuerte componente vocacional que los hace sentirse útil en la sociedad y que puede inducir a la auto realización y satisfacción personal", pero por el otro lado está "la existencia de aspectos negativos sobre la docencia, que puede llevar a desequilibrios personales y emocionales", esto debido a los sentimientos de frustración producto de que sus esfuerzos y su trabajo no se ven reflejados en la consecución de logros claros $y$ tangibles en un futuro inmediato.

Con relación al tipo de actividades que realiza el docente universitario, se encontró que el 30\% efectúa actividades académicas, administrativas y de orientación individual. Esto indica que el docente tiene a su cargo diversas funciones y responsabilidades al mismo tiempo, exigiéndole realizar acciones repetitivas de planeación, programación y ejecución relacionadas con la praxis educativa e investigativa. Dichas actividades exigen del docente gran dedicación y compromiso constante para evaluar y reestructurar el diseño metodológico del plan de curso y la pedagogía empleada para generar espacios dialógicos y de co-construcción social del conocimiento, en el que se indaga, comprende e interpreta la realidad, con el fin de hacer propuestas que permitan transformar la sociedad (Parra,1999). Desde luego, es una responsabilidad que cae sobre sus hombros, haciendo cada vez 
más compleja su labor, dado que debe ajustarse a los frecuentes cambios de los sistemas sociales, culturales, políticos, económicos, filosóficos, éticos y religiosos, por ende, debe preguntarse cómo están funcionando sus estrategias de enseñanza, qué tan eficaces son y cuál es el verdadero resultado que se está logrando en los estudiantes (Henao, Núñez y Quimbayo, 2010). En este sentido, el docente resulta inmerso en su labor formativa, dedicando la mayor parte de su vida en el fortalecimiento del ejercicio pedagógico, convirtiéndose, de este modo, en un posible factor de riesgo determinante para el desarrollo del estrés. Según los resultados obtenidos en este estudio se observó que los docentes dedican semanalmente a sus empleos principales de 31 a 60 horas (70\%), de 1 a 30 horas (20\%) y de 61 horas en adelante (10\%). Tales profesionales no solamente se dedican al oficio de la docencia, sino que además ejercen la psicología clínica en sus consultorios particulares. Durante mucho tiempo la docencia es considerada como una de las profesiones en las que más se experimenta estrés laboral, según lo corrobora una investigación pionera realizada por Chakravorty (1989; citado por Moriana y Herruzo, 2004: 599), quien afirmaba que "un $77 \%$ de las bajas de larga duración eran debidas a trastornos mentales en una muestra de 1.500 profesores con baja laboral". No solo por el tiempo que se invierte para el desarrollo académico en la planeación y ejecución de las clases, calificación de trabajos, preparación y calificación de las pruebas evaluativas, lograr que los estudiantes cumplan con la normatividad del centro educativo (teniendo en cuenta que muchos de estos son adolescentes con problemas para acatar las normas y seguir instrucciones), la ubicación del plantel educativo en una zona rural, urbana o marginal, lo cual afecta de manera significativa el equilibrio emocional de los docentes y la comunidad universitaria en general (Byrne, 1999; Leithwood, Jantziy Steinbach, 2001; Valero, 1997; citados por Morianay Herruzo, 2004). Por otro lado, los docentes afirmaron que alternamente ejercen la clínica psicológica en sus consultorios particulares, donde el $20 \%$ dedica entre 4 y 5 horas semanales a atender a sus pacientes, y otro $10 \%$ dedica 8 horas, frente a un $50 \%$ que manifestó no tener consultorio propio. En efecto, el ejercicio psicoterapéutico constituye otro de los factores de riesgo para experimentar estrés, debido a que es inherente para el profesional vivenciar un compromiso emocional al contemplar el sufrimiento de otros. En la relación terapeuta-paciente es frecuente que exista síntomas de irritabilidad y enojo, sentimientos de frustración, actitudes suspicaces, fatiga psicológica, síntomas psicosomáticos, como cansancio físico, dificultades respiratorias, problemas gastrointestinales, baja satisfacción laboral y alteraciones en el clima familiar como consecuencia del estrés prolongado producido por el quehacer psicológico que requiere tratar con personas que acuden al psicólogo para que le oriente en la resolución de sus problemas y dificultades, lo que en últimas se convierte en una carga emocional para el psicoterapeuta.

Otro aspecto es la calidad del sueño en los docentes, ya que este es un fuerte predictor del estrés laboral. En este caso, el $80 \%$ de los docentes reportaron que duermen diariamente entre 5 y 6 horas aproximadamente, el $10 \%$ de 3 a 4 horas y otro $10 \%$ de 1 a 2 horas. De acuerdo con esto, resulta preocupante debido a que los docentes no descansan lo suficiente durante la noche para iniciar sus labores al día siguiente, generando mayor desgaste en el funcionamiento cerebral y sus procesos, como lo es la percepción, la atención, memoria, pensamiento, lenguaje, motivación y emoción, así como las alteraciones en el ritmo cardiaco (Casaset al., 2002). Asimismo, el tiempo que dedican los docentes a sus familias diariamente compone otro factor predictor del estrés. Los docentes manifestaron que dedican a sus familias entre 1 y 2 horas (40\%), 3 y 4 horas (30\%), 5 y 6 horas (10\%), y no dedican tiempo (20\%). La última vez que realizaron un viaje familiar fue hace un mes $40 \%$, varios meses $30 \%$, menos de un año $10 \%$, más de un año $20 \%$. Estos resultados permiten evidenciar que aunque, cuentan con poco tiempo para compartir con su familia debido a las diversas exigencias laborales, hacen todo su esfuerzo para compartir con ellos. Sin embargo, es importante este aspecto, ya que puede ser un indicador de adicción y 
dependencia al trabajo, en la medida en que el empleado pasa más tiempo del requerido inmerso en actividades netamente laborales, por lo que es imposible para él sacar espacios de esparcimiento y diversión con sus seres queridos, debido a que experimenta ideas obsesivas y sufrimiento al no poder practicar las acciones repetitivas, así como sentimientos de culpa al realizar otras actividades que no se relacionen con su trabajo (Fernández y Echeburúa; citados por Alcóveret al., 2012).

Finalmente, para comprender las causas que originan el estrés laboral en docentes es necesario revisar factores multidimensionales, como son las características de personalidad susceptibles al estrés, alteraciones en las relaciones interpersonales porfalta de apoyo decompañeros, supervisores y administrativos, el desarrollo de la actividad laboral en organizaciones estructuradas bajo políticas burocráticas profesionalizadas, que basan su administración en la estandarización de las habilidades de sus miembros, frenando la autonomía en procura de una gestión que transforme y mejore los procesos académicos, investigativos y administrativos en el contexto universitario (Peiró,1992). Por último, el entorno socioprofesional, alberga cambios frecuentes en los procedimientos, tareas, funciones y roles en los programas de formación de los profesionales, debido a las variaciones en las necesidades de la población atendida.

\section{Conclusiones}

Los docentes universitarios que participaron en esta investigación presentan estrategias de afrontamiento ante el estrés, orientadas especialmente a la solución del problema. Esto lo evidencian las puntuaciones de las escalas del $\mathrm{CAE}$, como lo son la focalización en la solución del problema, reevaluación positiva y búsqueda de apoyo social, indicando que los docentes poseen recursos adaptativos y funcionales para atenuar sus dificultades y desafíos en la profesión como psicólogo y docente al mismo tiempo, ambas consideradas profesiones con alto componente de riesgo psicosocial en el desarrollar estrés laboral. Pese a que la correlación entre la variable de somatización y las variables del CAE fueron bajas, sí es importante prestar atención a los docentes que manifestaron estar experimentando sintomatología relacionada con el trastorno de somatización, ya que algunos mecanismos de afrontamiento no están siendo lo sufrientemente efectivos para enfrentar las situaciones estresantes en el ámbito personal, familiar y laboral. Por tanto, es necesario generar propuestas orientadas a fortalecer en docentes habilidades intra e interpersonales que le permitan comunicar asertivamente los aspectos académico-administrativo en el sitio de trabajo. Además, se debe visibilizar la importancia de la coherencia que debe existir entre las condiciones laborales que establecen las universidades en sus reglamentos, así como las directrices que imparten los funcionarios administrativos. También, habría que revisar las funciones y la carga laboral asignada a los docentes de tiempo completo, con el fin de garantizar mejores condiciones de trabajo, teniendo como base los principios de equidad y proporcionalidad para todos los miembros del equipo docente. De igual forma, se debe tener en cuenta que para la prevención del estrés laboral los docentes deben generar estrategias personales como la reevaluación positiva de los hechos y los acontecimientos, evitando creencias irracionales en la realidad, haciéndola ver agobiante y aterradora; la búsqueda de apoyo social en familiares, amigos y compañeros de trabajo para encontrar en conjunto soluciones alternativas a los problemas; mantener una actitud positiva frente a las situaciones desafiantes de la vida, realizando actividades lúdicas, deportivas y de contacto social que lo alejen de pensamientos negativos; compartir tiempo con seres queridos, optimizando el tiempo libre del que se dispone para asegurar su bienestar físico y psicológico, ya que este se verá reflejado en su labor docente y en las demás áreas que lo componen como ser humano. 


\section{Referencias}

Alcóver, C.;Moriano, J.;Osca, A.;Topa, G. (2012).Psicología del trabajo, Universidad Nacional de Educación a Distancia, Madrid, disponible en: http://books.google.es/oks?hl=es\&l$r=\& i d=I I Q d G 1 \cup M I d w C \& o i=f n d \& p-$ $\mathrm{g}=\mathrm{PA} 253 \& \mathrm{dq}=$ adicci\%C3\%B3n+al+trabajo\&ots=7bnVVfwTuD\&sig=qq_9DOt0rZn0j6y99L_om6zRvXE\#v=onepage\&q=adicci\%C3\%B3n\%20al\%20trabajo\&f=false

Atance, J. (1997). "Aspectos epidemiológicos del síndrome de burnout en personal sanitario", Revista Española de Salud Pública, núm. 71, vol. 3,disponible en: http://scielo.isciii. es/scielo.php?pid=\$1135-5727199700030 0008\&script=sci_arttext

Carrasco, Y.; Patricio, R. (2012). "Identificación del burnout en profesionales de la salud y factores relacionados con este fenómeno en el Hospital General Provincial Docente Riobamba y en el área de salud No. 6 Guano Penipe de la ciudad de Riobamba durante el periodo mayo-diciembre de 2012", Universidad Técnica Particular de Loja, disponible en: http://dspace.utpl.edu.ec// handle/123456789/5289

Carrillo, R.; Gómez, K.; Espinosa, I. (2012). “Síndrome de burnout en la práctica médica", Revista Medicina Interna de México, núm. 8, vol. 6, pp. 580-582, disponible en: http:// www.medigraphic.com/pdfs/medintmex/ mim-2012/mim126j.pdf

Casado, D.(2000). "Modelo de afrontamiento de Lazarus como heurístico de las intervenciones psicoterapéuticas", Equipo de Salud Mental Alcalá-Dos Hermanas, Hospital El Tomillar, disponible en: http://www.cop.es/delegaci/ andocci/files/contenidos/VOL20_3_5.pdf

Casas, J.; Repullo, J.; Lorenzo, S.; Cañas, J. (2002). "Dimensiones y medición de la calidad de vida laboral en profesionales sanitarios", disponible en: http://www.dinarte.es/ras/ ras23/pdf/administracion2.pdf

Contreras, F.; Esquerra, G.; Espinosa, J.; Gómez, V. (2007) "Estilos de afrontamiento y calidad de vida en pacientes con insuficiencia renal crónica (IRC) en tratamiento de hemodiálisis", Acta Colombiana de Psicología, vol.,10, núm. 2, pp. 169-179, disponible en: http://portalweb.ucatolica.edu.co/easyWeb2/acta/pdfs/v10n2/art-15_169-179. pdf

Contreras, F.; Espinosa J.; Esquerra, G. (2009). “Personalidad y afrontamiento en estudiantes universitarios", Revista Univ. Psychol., vol. 8, núm. 2, pp. 311-322, disponible en:

http://www.scielo.org.co/pdf/rups/v8n2/ v8n2a02.pdf

Domínguez, F. (2004). "Riesgo psicosocial en la universidad: estresores propios del docente universitario", Revista Salud de los Trabajadores, vol. 2, núm. 2, julio, Universidad de Huelva.

DSM IV-TR. Manual diagnóstico y estadístico de los trastornos mentales, Barcelona: Ed. Manson.

Federación de Enseñanza CC.OO. de Andalucía (2012). Temas para la educación. Revista digital para Profesionales de la enseñanza, núm. 21.

Fernández, N. (2006).Características de desarrollo psicológico del adulto, Universidad Nacional Autónoma de México, disponible en: http://www.econtinua.com/documentos/ desarrollo\%20adultez.pdf

Flores, C.; Huerta, R.; Carrillo, J.; Zarate, T.; Bernal, M.; Morales, I. (2009). "Incidencia de estrés en odontólogos de diferentes especialidades ocasionado por ruido en el consultorio dental", Revista Nova Scientia, vol. 1, núm. 2, pp. 1-21, Universidad de La Salle. 
Gil-Monte, P. (2003). “El síndrome de quemarse por el trabajo (síndrome de burnout) en profesionales de enfermería", Revista EletrónicalnterAçãoPsy, vol. 1, núm. 1, pp. 19-33, Universidad de Valencia, disponible en: http://www.bvsde.ops-oms.org/bvsacd/cd49/artigo3.pdf

Gil-Monte, P.; Peiró, J. (1997). Desgaste psíquico en el trabajo: el síndrome de quemarse, Madrid: Síntesis.

Gonzales, E.; Gutiérrez, R. (2006). "La carga de trabajo mental como factor de riesgo de estrés en trabajadores de la industria electrónica", Revista Latinoamericana de Psicología, disponible en: http://www.scielo. org.co/scielo.php?pid=S0120-053420060 00200003\&script $=$ sci_arttext

González, M.; Landero, R. (2008). “Confirmación de un modelo explicativo del estrés y de los síntomas psicosomáticos mediante ecuaciones estructurales", Rev. Panam. Salud Pública, vol. 23, núm. 1, pp. 7-18, disponible en: http://www.scielosp.org/pdf/ rpsp/v23n1/a02v23n1.pdf

Guerrero, E. (2003).“Análisis pormenorizado de los grados de burnout y técnicas de afrontamiento del estrés docente en profesorado universitario", Revista Anales de Psicología, vol. 19, núm. 1, pp.145-158, Universidad de Extremadura.

Guzmán, R. (2011). “Trastorno por somatización: su abordaje en atención primaria", Especial. Rev. Clínica de Medicina de Familia, disponible en: http://www.revclinmedfam. com/articulo.php?art=227

Henao, A.; Núñez, M.;Quimbayo, J. (2010). “EI rol del profesional de la salud como docente universitario", Universidad de la Sabana,Aguichán, disponible en: http:// aquichan.unisabana.edu.co/index.php/ aquichan/article/viewArticle/1604/2122
Mamani, A.; Obando, R.; Uribe, A.; Vivanco, M. (2007). "Factores que desencadenan el estrés y sus consecuencias en el desempeño laboral en emergencia", Revista Peruana de Obstetricia y Enfermería, vol. 3,núm. 1, disponible en:http://revistas.concytec.gob. pe/scielo.php?pid=S1816-771320070001 00007\&script=sci_arttext

Marulanda, I. (2007). “Estrés laboral enemigo silencioso de la salud mental y la satisfacción con la vida", documento de trabajo, Universidad de los Andes, Facultad de Ciencias Sociales, Departamento de Psicología. Bogotá.

Matud, M.; García, M.; Matud, M. (2002). “Estrés laboral y salud en el profesorado: un análisis diferencial en función del género y del tipo de enseñanza", Revista Internacional de Psicología Clínica y de la Salud, vol.2, núm. 3, pp. 451, disponible en: http://www. aepc.es/ijchp/articulos_pdf/ijchp-50.pdf

Moreno, B.; Garrosa, E.; Rodríguez, R. (2009). “El burnout del profesorado universitario y las intenciones de abandono: un estudio multi-muestra", documento interno, Universidad de Zaragoza, Universidad Autónoma de Madrid, Universidad de Barcelona, disponible en: https://docs. google.com/viewer?url=http://www.redalyc.org/pdf/2313/231316498005.pdf\&chrome $=$ true

Moriana, J.; Herruzo, J. (2004).“Estrés y burnout en profesores", Revista International Journal of Clinical and Health Psychology, vol.4, núm. 3, pp. 597-621, disponible en:http://www. aepc.es/ijchp/articulos_pdf/ijchp-126.pdf

Ortega, C.; López, F. (2004). “El burnouto síndrome de estar quemado en los profesionales sanitarios: revisión y perspectivas", Universidad de Almería, España disponible en: http://www.aepc.es/ijchp/articulos_pdf/ ijchp-100.pdf 
Palmer, Y.; Gómez, A.; Cabrera, C.; Prince, R.; Searcey, R. (2005).“Factores de riesgo organizacionales asociados al síndrome de burnout en médicos anestesiólogos", Rev. Medigraphic Artemisa. Salud Mental, vol. 28, pp. 88, disponible en: http://www. medigraphic.com/pdfs/salmen/sam2005/sam051i.pdf

Pando, M.; Aranda, C.; Guadalupe, M.; Flores, E.; Pozos, E. (2006). "Factores psicosociales y burnout en docentes del centro universitario de ciencias de la salud", Revista Medigraphic Artemisa, vol. VIII, núm. 3, recuperado de: http://www.medigraphic.com/ pdfs/invsal/isg-2006/isg063f.pdf

Parra M. (1999). "Generalidades de la evaluación”, Revista de Medicina, núm. 47, vol. 4, Universidad Nacional de Colombia.

Peiró, J.M. (1992). Desencadenantes del estrés laboral, Madrid: Eudema.

Ponce, C.; Bulnes, M.; Aliaga, J.; Atalaya, M.; Huertas, R. (2005). “Síndrome del quemado por estrés laboral asistencial en grupos de docentes universitarios", Revista IIPSI, vol. 8, núm. 2, pp. 87-112, Facultad de Psicología UNMSM.

Quintanilla, M. (2004). “Prevalencia del síndrome de burnout en las enfermeras de la Unidad de Paciente Crítico del Hospital del Trabajador Santiago de Chile y una propuesta de intervención", Revista Chilena de Medicina Intensiva,vol.19, núm. 1, pp. 33-38, dis- ponible en: http://www.revistamedintensiva.cl/pdf/11Quintanilla.pdf

Sánchez, M.; Mardones, R. (2010). “El lugar del psicólogo educacional en el intrincado camino entre el profesional y la escuela: una visión interpretativa desde dos experiencias prácticas", Revista Científica de Psicología, Ciencias Sociales, Humanidades y Ciencias de la Salud, vol. 2, núm. 1, pp.7185, disponible en: http://www.dialogos. unsl.edu.ar/files/el_lugar_del_psicologo_ educacional_en_el_intrincad.pdf

Solis, C.; Vidal, A. (2006) "Estilos y estrategias de afrontamiento en adolescentes", Revista de Psiquiatría y Salud Mental Hermilio Valdizan, vol. 7, núm. 1, pp. 33-39, disponible en:http://www.hhv.gob.pe/revista/2006/3\%20ESTILOS\%20Y\%20ESTRATEGIAS\%20DE\%20AFRONTAMIENTO.pdf

Valadez, A.; Bravo, M.; Vaquero, J. (2011).“Estrategias de afrontamiento empleadas por docentes universitarios", Revista Electrónica de Psicología Iztacala, vol. 14, núm. 1, disponible en:http://revistas.unam.mx/index. php/repi/article/view/24794.

Vega, N.; Sanabria, A.; Domínguez, L.; Osorio, C.; Bejarano, M. (2009). "Síndrome de desgaste profesional en cirujanos colombianos", Revista Colombiana de Cirugía, vol. 24, pp.153-64,disponible en: http://www. ascolcirugia.org/revista/revistajulioseptiembre2009/Desgaste\%20153-164.pdf 
